\title{
Efficacy control of therapy using circulating epithelial tumor cells (CETC) as "Liquid Biopsy": trastuzumab in HER2/neu-positive breast carcinoma
}

\author{
Katharina Pachmann - O. Camara • \\ T. Kroll • M. Gajda · A. K. Gellner • \\ J. Wotschadlo $\cdot$ I. B. Runnebaum
}

Received: 1 June 2011/Accepted: 20 June 2011/Published online: 8 July 2011

(C) The Author(s) 2011. This article is published with open access at Springerlink.com

\begin{abstract}
Purpose The majority of targeted personalized cancer therapies are effective only in part of the patients, and most of these drugs are excessively expensive. Therefore, methods are urgently required, which reveal already early during treatment, whether the therapy is effective. In the present report, monitoring of circulating epithelial tumor cells (CETC) was used as a timely control of trastuzumab therapy in patients with HER2/neu-positive breast cancer. Methods Seventy-nine sequential HER2/neu-positive breast cancer patients, 35 without trastuzumab, and 36 treated with 1 year of trastuzumab treatment were included. CETC from unseparated white blood cells stained with FITC-anti-EpCAM were analyzed repeatedly during chemotherapy and between 2 and 10 times during 1 year of maintenance treatment or observation.

Results Patients treated with trastuzumab had a better relapse-free survival than patients without trastuzumab treatment during the first 2-4 years of follow-up. Decrease in numbers or no change versus highly variable numbers or increase (fivefold or more) allowed to discriminate highly significantly and clearly $(P<0.0001$, hazard ratio 5.5$)$
\end{abstract}

K. Pachmann ( $₫) \cdot$ T. Kroll · A. K. Gellner · J. Wotschadlo Department of Experimental Hematology and Oncology, Clinic for Internal Medicine II, Friedrich Schiller University, Jena, Erlanger Allee 101, 07747 Jena, Germany

e-mail: Katharina.pachmann@med.uni-jena.de

O. Camara · I. B. Runnebaum

Women's Hospital, Friedrich Schiller University, Jena, Jena, Germany

M. Gajda

Institute of Pathology, Friedrich Schiller University,

Jena, Jena, Germany between patients with a low or high risk of relapse. An increase in CETC was accompanied by an increasing portion of cells containing a very high number of HER2/ neu gene amplificates.

Conclusions Analysis of the behavior of CETC can, in the future, contribute to evaluate the efficacy of targeted therapy early during the course of the disease, sparing patients unnecessary treatment but also to reduce the costs for the health system and to downsize the extent and length of clinical studies.

Keywords HER2/neu-positive breast cancer · Circulating epithelial tumor cells - Trastuzumab therapy - Cancer therapy

\section{Purpose}

The majority of targeted personalized cancer therapies have, so far, failed to demonstrate convincing efficacy, and even the few active agents, the most successful of which is trastuzumab, are effective only in part of the patients carrying the respective targets. In $20-25 \%$ of invasive breast cancers, the ErbB2 growth factor receptor is overexpressed mainly due to an amplification of the HER2/neu gene and patients with this amplification have an inferior prognosis. The insight into the molecular mechanisms of this genetic aberration allowed the development of a number of molecules directed to the ErbB2 receptor such as the humanized antibody trastuzumab, a monoclonal antibody aimed at the extracellular juxtamembrane domain of HER2/neu (O'Donovan and Crown 2007), which enabled one of the first targeted therapies in combination with standard adjuvant therapy, thus decreasing the risk of recurrence (Hudis 2007; Olopade et al. 2008). 
Although our understanding of the molecular events that underlie tumor development including gene expression profiling has improved treatment, only part of those patients, carrying the HER/2neu overexpression, respond to the targeted therapy and a considerable number of patients would farewell also without trastuzumab $(77 \%$ at 2 years observation; Piccart-Gebhart et al. 2005), To date, statistical methods taking several years before results are available are still the only manner of determining the efficacy of the applied treatment, and later investigations often do not confirm initial results. Thus, Spielmann et al. (2009) presented a 3-year follow-up of the phase III PACS04 trial where the difference in disease-free survival between the observation arm and the trastuzumab arm in contrast to the previously published difference of $8.4 \%$ from the Hera study (Piccart-Gebhart et al. 2005) nearly disappeared $(73.2 \%$ vs. $72.7 \%)$. This shows that even with time-consuming studies it is difficult to assure the benefit of a new therapy in medical trials. Most of these drugs are excessively expensive straining the health systems of the developed countries and being prohibitive in the developing world. In addition, a high proportion of patients may farewell also without trastuzumab and prediction for the individual patient is restricted. Therefore, methods are urgently required, which can determine early on whether and how patients respond to the applied therapy and which can evaluate the individual patient's response to treatment earlier and continuously.

One approach to gauge the specific response to therapy in the individual patient is monitoring the size reduction in the tumor during primary (neoadjuvant) therapy. In previous reports, the importance of circulating tumor cell monitoring (Pachmann et al. 2001, 2005a) in peripheral blood for determination of chemotherapy efficacy in conjunction with neoadjuvant therapy had already been demonstrated. We have shown that circulating tumor cells in the neoadjuvant setting respond to therapy in a way identical to the tumor (Pachmann et al. 2005b). However, a good response to neoadjuvant treatment does not necessarily translate into better prognosis in biologically more aggressive breast cancers (Darb-Esfahani et al. 2009). Indeed, after the initial reduction during neoadjuvant chemotherapy, we reported a subsequent massive dissemination of cells from the shrinking tumor (Camara et al. 2007), which are spread via peripheral blood and, if not treated adequately, can grow into metastases (Staal et al. 2009).

We were able to show that the response of CETC to chemotherapy is also applicable for monitoring therapy response in the adjuvant situation and that this is highly correlated to relapse-free survival (Pachmann et al. 2008).

We had prospectively analyzed patients with HER2/neupositive breast cancer for their behavior of circulating tumor cells without trastuzumab before trastuzumab was included into the guidelines of treatment for HER2/neu breast cancer, and these patients were used as the control population, whereas at present a control population of patients with this disease deprived of trastuzumab will no longer be available because of ethical reasons.

In the present work, the impact of trastuzumab on CETC in peripheral blood after adjuvant chemotherapy was longitudinally monitored, quantifying the number of these cells during or without adjuvant trastuzumab therapy in individual HER2/neu-positive patients and their HER2/neu amplification status in order to investigate whether it is thus possible to assess the efficacy of this treatment and its correlation to clinical outcome.

\section{Methods}

Patients and treatment

Seventy-nine sequential patients were included in the study. All patients consented with written informed consent to blood drawing at the onset of treatment according to ethics committee approval. (Approved by the local ethics committee. Ethik Kommission der Friedrich Schiller Universität Jena an der Medinischen Fakultät, Jena, Germany). Clinical data were collected from the unit's database and updated periodically. HER2/neu status was routinely determined immunohistochemically, and in cases of doubt, additionally verified by FISH. 35 patients diagnosed as positive (IHC $3+$ ) had not received adjuvant trastuzumab between September 2001 and September 2005 representing the control group. 36 patients overexpressing HER2/neu received adjuvant chemotherapy followed by 1 year trastuzumab between 2005 and February 2008. 8 patients were primarily metastasized and received chemotherapy together with trastuzumab. Adjuvant chemotherapies applied were epirubicin/cyclophosphamide (EC) with or without taxane, fluorouracil/epirubicin/cyclophosphamide (FEC) with and without taxane, fluorouracil/epirubicin/cyclophosphamide (FEC)/taxane/gemcitabine, and cyclophosphamide/methotrexate/fluorouracil (CMF). All estrogen receptor (ER) positive patients were administered hormone therapy after adjuvant chemotherapy.

\section{CETC analysis}

About 2-7 ml of blood anticoagulated with EDTA was drawn at each visit before and during chemotherapy and during 1 year of trastuzumab maintenance therapy or follow-up at least twice and up to 10 times. In order to adjust for shipping delays, all samples were analyzed $48 \mathrm{~h}$ after blood drawing including exclusively viable cells into the analysis. $1 \mathrm{ml}$ of blood was lysed with ammonium chloride 
(Qiagen, Hilden, Germany). For detection of CETC, white cells from the sediment were subject to MAINTRAC ${ }^{\circledR}$ analysis, omitting all enrichment steps, diluting the pellet in $500 \mu \mathrm{L}$ of phosphate-buffered saline (pH 7.4) and adding $12.5 \mu \mathrm{L}$ of fluorescein isothiocyanate-conjugated mouse antihuman epithelial antibody (EpCam) (Miltenyi Biotec, Bergisch Gladbach, Germany) and, optionally, $5 \mu \mathrm{L}$ of phycoerythrin-labeled CD45 (Miltenyi Biotec) simultaneously for $15 \mathrm{~min}$ in the dark. Propidium iodide (PI) or 7AAD was added for recording dead cells. Analysis of red and green fluorescence of the cells was performed using a laser scanning cytometer (LSC ${ }^{\circledR}$ Compucyte Corporation, Cambridge, MA), enabling relocation of cells for visual examination of vital epithelial cells (which were always CD45 negative) as extensively described in a previous study (Pachmann et al. 2005a) or image analysis using a ScanR ${ }^{\circledR}$ microscope (Olympus, Hamburg, Germany). For LSC $^{\circledR}$ measurements, a defined volume of the cell suspension was applied to a defined area on an adhesion slide (Menzel Gläser, Braunschweig, Germany) and measurements were performed on this area. Cells were detected by their forward scatter and red and green fluorescence recorded (Pachmann et al. 2005a). CD45 staining contributed to better separate brightly staining lymphocytes and faintly staining granulocytes from the CD45-negative epithelial cells that were selected and gated by their green fluorescence and relocated and analyzed for vitality by propidium iodide exclusion. Morphological analysis had already been performed previously, and a fluoromicrograph of a typical group of four epithelial antigen-positive cells (taken from Pachmann et al. 2001) is shown in Fig. 1a. Three of the cells display tiny caps, whereas the fourth exhibits strong staining, which was considered as intracellular staining. Indeed, hematoxylin-eosin staining (HE) in Fig. $1 \mathrm{~b}$ reveals that this cell is a dying cell. In addition, a normal epithelial antigen-negative leukocyte is visible only in HE staining. In all further analyses, vitality was analyzed by PI or 7AAD exclusion. Only vital cells were counted. For ScanR ${ }^{\circledR}$ image analysis, defined volumes of cell suspension were added to glass bottom microtiter plate wells and analysis began when the cells had settled on the well bottom. Figure 1c shows a fluoromicrograph including two vital epithelial antigen-positive cells with typical green caps, adjacent to two faintly CD45-stained granulocytes and a bright CD45-stained vital lymphocyte in addition to a dead PI-positive cell. In Fig. 1d, an overlay of a fluoromicrograph with transmitted light is shown. Note, the red 7AAD-positive fluorescing nucleus of a dead normal leukocyte in the neighborhood of the green fluorescing CETC and a red and green fluorescing dying CETC. Results from both microscope analyses were highly comparable. Sequential analyses for individual patients were always performed on the same instrument. Numbers of live CETC were calculated per ml. As seen from Fig. 3, CETC numbers were already strongly influenced by chemotherapy and varied before the start of trastuzumab therapy between 100 and a maximum of 30,000. These values are much higher than those reported earlier (Lobodasch et al. 2007) in primary breast cancer and as detected by the CellSearch approach (Cristofanilli et al. 2004) in metastatic breast cancer due to omission of magnetic separation because magnetic separation may lead to an extensive loss of specific cells (Pachmann et al. 2005a) as supported by a recently published report (Coumans et al. 2010). Previous studies with our approach had revealed $97 \%$ specificity and 92\% sensitivity (Pachmann et al. 2005a), and analysis of 25 patients with hematological malignancies did not show any EpCam-positive cells in any of the patients whether with full blown leukemia, in complete remission with regenerating hematopoiesis, or in relapse (Pachmann et al. 2005b). In the longitudinal analysis during therapy, a continuous reduction or increase of fivefold or more over the observation time of 1 year was defined as a significant decrease or increase. In situ hybridization was subsequently performed in selected patients.

\section{FISH (fluorescence in situ hybridization) analysis}

About $100 \mu$ of unstained cell suspension were prepared as described above. $3 \mu \mathrm{l}$ of FITC-HEA were added followed by cold incubation for $15 \mathrm{~min}$. No PE-CD45 (orange) was used in order to avoid interference with the fluorescent HER2-DNA probe (red).

The cell suspension was then diluted with $10-900 \mu \mathrm{l}$ of PE buffer depending on the assessed CETC density. An equal volume of BSA $4 \%$ was added. The marked area on the slide comprised about $1 \mathrm{~cm}^{2}$. The slides were then airdried for at least $8 \mathrm{~h}$ in a dark place. Storage up to 3 months before further processing was possible without loss of quality if kept in the dark. The FISH analysis was performed using Path Vision HER2 DNA probe kit (LSI HER-2/CEP17 probe, Abbott GmbH and Company, KG, Wiesbaden-Delkenheim, Germany). The manufacturer's protocol was adapted to a fixed cell suspension instead of paraffin-embedded tissue. Fixation of the cells was achieved by incubating the slide in paraformaldehyde for $10 \mathrm{~min}$ followed by treatment with Protease K. After incubation in post-hybridization washing buffer (2X SSC and $0,3 \% \mathrm{NP}-40$ ) for $5 \mathrm{~min}$, denaturation was performed in heated formalin at $72^{\circ} \mathrm{C}$ for $5 \mathrm{~min}$ followed by dehydration in ethanol at 70,85 , and $96 \%$ for 1 min each. $1.5 \mu$ of the denaturated DNA probe, including a HER2 DNA probe labeled with SpectrumOrange and a CEP17 alpha DNA probe labeled with SpectrumGreen, was used for hybridization with incubation for $14-18 \mathrm{~h}$ at $37^{\circ} \mathrm{C}$ in a humidified chamber. Then, slides were washed with post-hybridization 

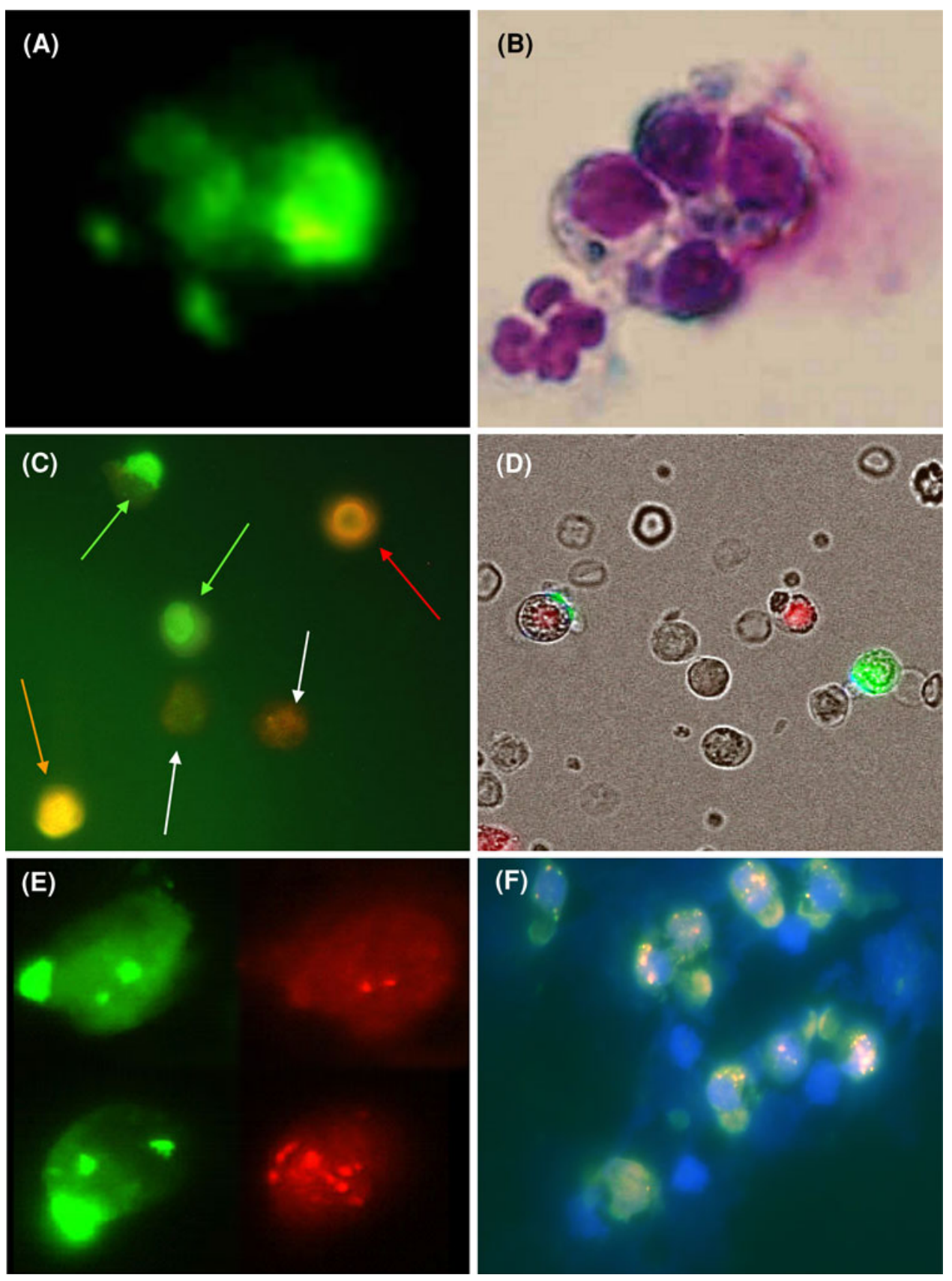

Fig. 1 a Fluoromicrograph of a typical group of four epithelial antigen-positive cells (taken from Darb-Esfahani et al. 2009). Three of the cells display tiny caps, whereas the fourth exhibits strong staining, which was considered as intracellular staining. b Hematoxylin-eosin staining $(H E)$ reveals that the intracellularly stained cell is a dying cell. In addition, a normal epithelial antigen-negative leukocyte is visible only in HE staining. c Fluoromicrograph of epithelial cells with green fluorescence, exclusively surface located including two vital epithelial antigen-positive cells with typical green caps (green arrows), adjacent to two faintly CD45-stained granulocytes (white arrows) and a bright CD45 stained vital lymphocyte (red arrow) in addition to a dead PI-positive cell (orange arrow). d Overlay of a fluoromicrograph with transmitted light. Note, the red 7AAD-positive fluorescing nucleus of a dead normal leukocyte in

the neighborhood of the green fluorescing CETC and a red and green fluorescing dying CETC. e Two CETC from the same patient using the green and red filter, respectively. In both cells, the typical green cap of the EpCam, and in addition, the two green signals of the CEP17 alpha DNA probe can be identified. The upper cell carries only two HER2/neu signals, whereas in the lower cell more than 10 signals can be detected. f Fluoromicrograph overlay of the HER2/neu FISH analysis of a group of tumor cells from a breast cancer patient: besides the normal blood cells (nuclear stain was DAPI, only the nuclei are visible), one can see a group of tumor cells staining with typical green caps with the FITC-labeled anti-EpCAM. These cells carry a varying quantity of HER2/neu amplifications (pink-red dots in the cell nucleus) 
washing buffer at room temperature for $1 \mathrm{~min}$ and subsequently at $72^{\circ} \mathrm{C}$ for $2 \mathrm{~min}$. After drying, 4,6-diamidino-2phenylindol (DAPI) was applied for counterstaining of the nuclei. An Axiovert fluorescent microscope (Zeiss Jena Germany) with suitable filters was used for cell analysis with a $100 \times$ oil immersion objective. Epithelial cells were identifiable by a typical green staining of the surface as mentioned above; DAPI staining confirmed integrity of the nucleus. Now, all the red signals within the nucleus were counted, which led to a classification of each cell as following: cells containing $2-5,6-10$, or $>10$ signals. The number of centromeres labeled by the CEP17 DNA probe was assessed. 20 consecutive CETC per sample were analyzed and the percentage of each class of signals was calculated.

According to the most recent recommendations by the ASCO (American Society of Clinical Oncology), cells with more than 6 signals were considered amplified. However, most previous researchers referred to $>4$ signals as HER2 amplification. In Fig. 1e, two CETC from the same patient are shown with the typical green cap of the EpCam and the two green signals of CEP17. The upper cell carries only two HER2/neu signals, whereas in the lower cell more than 10 signals can be detected. A fluoromicrograph of a group of cells comprising leukocytes and CETC is shown in Fig. 1f. The high variability in HER2/neu amplification in this cell group from one patient is obvious.

\section{Statistics}

For survival analyses, we used Kaplan-Maier plots and an analysis over strata and a Cox regression analysis of the SPSS statistical program (SPSS Inc, Chicago, IL). The discriminatory power of the analysis of the behavior of the circulating cells over time (decrease or increase) was high enough that a calculation assuming a power of $80 \%$ showed that the patient population necessary was reached (Sobrero and Bruzzi 2009).

\section{Results}

Table 1 gives an overview of the 79 consecutive patients treated with and without trastuzumab therapy at our institution. 20 patients have suffered relapse during the observation period: 15 in the group without adjuvant trastuzumab in part due to the longer observation time and 5 in the group receiving trastuzumab. Among the 8 patients with primary metastasized tumors, 5 progressed during the observation period. The baseline characteristics of the untreated and the trastuzumab-treated groups were well balanced with respect to tumor size, estrogen receptor expression, HER2/neu expression, and lymph node status with a somewhat higher frequency of anthracycline-based therapies in the trastuzumab-treated patients.

Kaplan-Meier relapse-free survival curves from the 35 patients without trastuzumab and the 36 patients treated for 1 year with trastuzumab are shown in Fig. 2. Although this was not a randomized study, outcome characteristics at 2 years were well comparable to the larger studies (Joensuu et al. 2006; Piccart-Gebhart 2006; Romond et al. 2005) and the difference in chemotherapeutic regimen could at most have contributed to the better outcome of the trastuzumab treatment group. However, our patients were observed another 4-6 years in a prospective follow-up beyond the time of 2 years, results which are still lacking from the NCT 00045032 trial. There was a trend to longer relapse-free survival in our restricted patient sample of HER2/neu-positive patients treated with 1 year sequential trastuzumab after chemotherapy compared to a patient sample not receiving trastuzumab therapy; however, this did not reach statistical significance (Fig. 2). The KaplanMeier plots indicate that the highest benefit occurred between years 2 and 3 after diagnosis. Thereafter, the curves tended to merge. But, even in the group not treated with trastuzumab, $50 \%$ of patients were still alive without relapse after 5 years. There was, however, no plateau and relapses occurred continuously. Patients with primarily metastasized tumors, although receiving trastuzumab simultaneously with chemotherapy, had the poorest prognosis.

Since lymph node involvement is a confirmed negative prognostic factor, we examined the influence of lymph node status in trastuzumab-treated and untreated patients. In the control group with no trastuzumab therapy, there was no significant difference between lymph node-negative patients and lymph node-positive patients, whereas under trastuzumab therapy HER2/neu-positive patients without lymph node involvement had a tendency to better prognosis than patients with positive lymph nodes $(P=0.113)$ (not shown).

The question arises; what is the effect of the antibody which is directed to the remnant systemic part of the tumor, the circulating tumor cells, and why is the benefit of trastuzumab limited and obviously only lasts for a restricted time?

In our institution, prior to the establishment of trastuzumab therapy, HER2/neu-positive patients were already being monitored prospectively for their numbers of CETC during chemotherapy treatment and the further course of disease. These patients now provided a unique source for further analyzing the factors contributing to relapse-free survival.

In almost all patients, CETC were detected before therapy and even after systemic chemotherapy still varying 
Table 1 Overview of the clinical data of patients analyzed for CETC without and with trastuzumab treatment
$H R$ hormone receptor, $C R$ complete remission, $N A$ not analyzed

\begin{tabular}{|c|c|c|c|c|c|}
\hline & Total & $\begin{array}{l}\text { Patients treated } \\
\text { without } \\
\text { trastuzumab }\end{array}$ & $\begin{array}{l}\text { Patients treated } \\
\text { with trastuzumab }\end{array}$ & $P$ & $\begin{array}{l}\text { Primarily } \\
\text { metastsized } \\
\text { patients }\end{array}$ \\
\hline & $79(100 \%)$ & $35(44 \%)$ & $36(46 \%)$ & & $8(10 \%)$ \\
\hline HR positive & $55(70 \%)$ & $23(66 \%)$ & $26(72 \%)$ & 0.434 & $6(75 \%)$ \\
\hline HR negative & $23(29 \%)$ & $12(34 \%)$ & $9(25 \%)$ & & $2(25 \%)$ \\
\hline NA & $1(1 \%)$ & $0(0 \%)$ & $1(3 \%)$ & & $0(0 \%)$ \\
\hline Age & 58 & 60 & 55 & & 60 \\
\hline HER2/neu-positive & $74(94 \%)$ & $33(94 \%)$ & $35(97 \%)$ & 0.307 & $6(75 \%)$ \\
\hline HER2/neu negative & $3(4 \%)$ & $1(3 \%)$ & $0(0 \%)$ & & $2(25 \%)$ \\
\hline NA & $2(2 \%)$ & $1(3 \%)$ & $1(3 \%)$ & & $0(0 \%)$ \\
\hline Tumor status $\mathrm{T} 1$ & $48(61 \%)$ & $22(63 \%)$ & $22(61 \%)$ & & $4(50 \%)$ \\
\hline Tumor status $T>1$ & $28(35 \%)$ & $11(31 \%)$ & $13(36 \%)$ & & $4(50 \%)$ \\
\hline NA & $3(4 \%)$ & $2(6 \%)$ & $1(3 \%)$ & & \\
\hline Lymph node status N0 & $42(53 \%)$ & $19(54 \%)$ & $20(55 \%)$ & 0.916 & $3(37 \%)$ \\
\hline Lymph node status N1 & $35(45 \%)$ & $15(43 \%)$ & $15(42 \%)$ & & $5(63 \%)$ \\
\hline NA & $2(2 \%)$ & $1(3 \%)$ & $1(3 \%)$ & & $0(0 \%)$ \\
\hline $\mathrm{CR}$ & $54(68 \%)$ & $20(57 \%)$ & $31(86 \%)$ & & $3(37 \%)$ \\
\hline Relapse & $25(32 \%)$ & $15(43 \%)$ & $5(14 \%)$ & 0.007 & $5(63 \%)$ \\
\hline \multicolumn{6}{|l|}{ CETC } \\
\hline Decrease & $46(58 \%)$ & $19(54 \%)$ & $25(69 \%)$ & & $2(25 \%)$ \\
\hline Increase & $26(33 \%)$ & $9(26 \%)$ & $11(31 \%)$ & & $6(75 \%)$ \\
\hline NA & $7(9 \%)$ & $7(20 \%)$ & $0(0 \%)$ & & $0(0 \%)$ \\
\hline
\end{tabular}

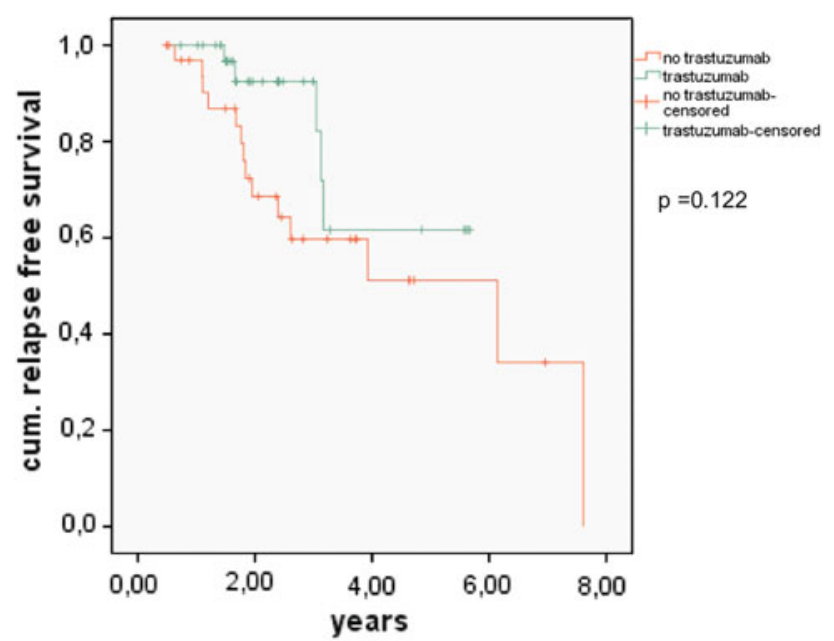

Fig. 2 Results from 36 HER2/neu-positive patients treated with 1 year sequential trastuzumab after chemotherapy (green line) as compared to a historical patient sample of 35 patients not receiving trastuzumab therapy (red line)

numbers of circulating tumor cells were detectable. Analysis of circulating tumor cells in blood is comparable to a snapshot; therefore, rather than individual values, the tendency of the alterations was parsed and the behavior classified as either decreasing/stable or highly variable and/ or increasing during the year of treatment with trastuzumab or, if relapse occurred earlier, until relapse. In patients without trastuzumab, the behavior of CETC during the same time was recorded. Figure 3 shows examples of representative courses of CETC. In Fig. 3a, typical courses during maintenance therapy with trastuzumab with decreasing CETC are shown and all of these patients are still in complete remission. Typical courses of CETC in patients with highly variable or increasing CETC are shown in Fig. 3b. Two of these patients have suffered relapse. CETC behavior without trastuzumab was comparable, but it should be mentioned that the highly variable saw-toothed characteristics were mainly seen in the trastuzumab treatment group. Figure $3 \mathrm{c}$, d show typical CETC courses in patients without trastuzumab treatment. All three of the patients with increasing CETC have already relapsed. In Fig. 3e, f, two patients are shown where decrease as well as increase in CETC during trastuzumab was accompanied by an increase or decrease in the fraction of cells with high amplificates of the HER2/neu gene, suggesting that response or resistance to therapy may be mainly due to this population of cells. This phenomenon is now being further investigated.

For some patients in the observation group, only single measurements were available, therefore increase/decrease in cell numbers could not be determined. Still, the difference in outcome between patients with decreasing and increasing/ variable numbers of CETC was highly significant both 
(A)

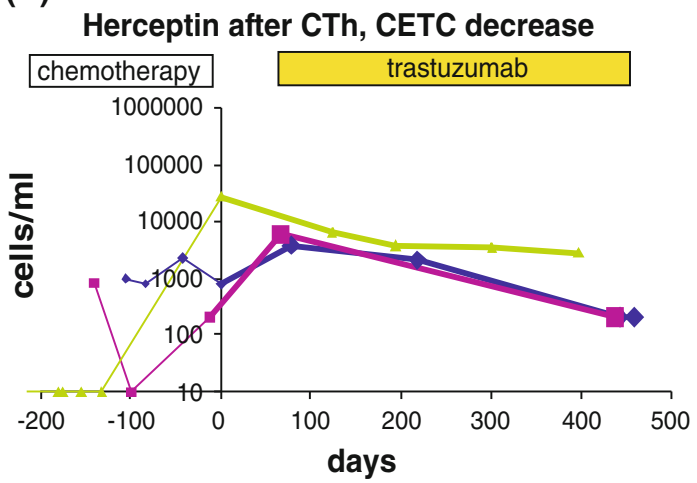

(C)

\section{No Trastuzumab after CTh, CETC decrease}

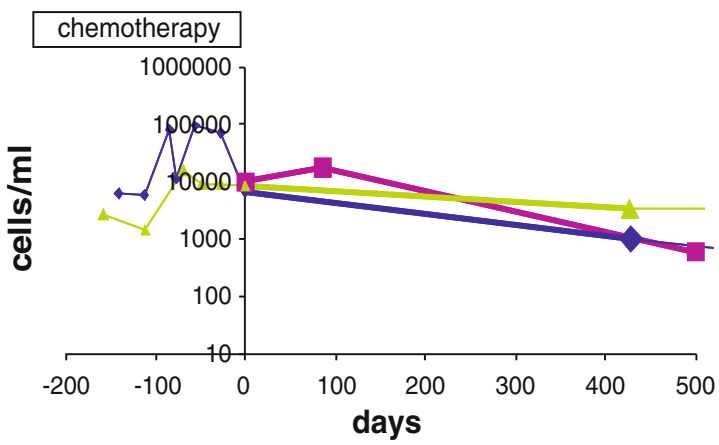

(E)

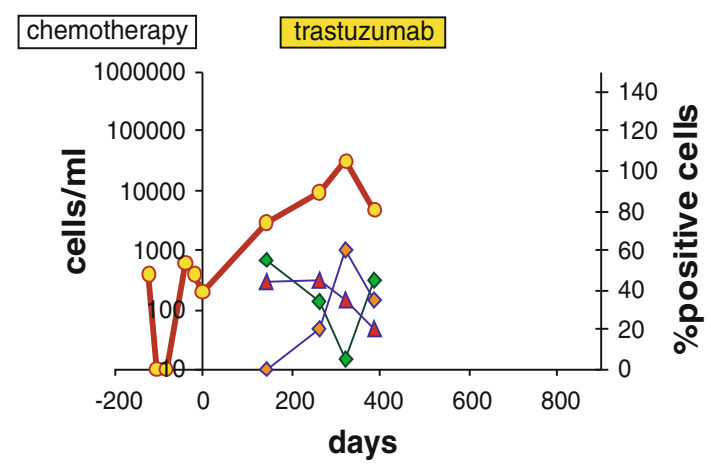

Fig. 3 Typical courses of CETC numbers from 3 individual patients each. Values on the negative $x$-axis are cell values during chemotherapy, while on the positive $x$-axis, the values during trastuzumab therapy are plotted. a Typical courses for three individual patients during chemotherapy followed by 1 year of trastuzumab decrease under trastuzumab with decreasing CETC. b Typical courses of CETC in patients during chemotherapy followed by 1 year of trastuzumab with highly variable or increasing CETC during

during maintenance therapy with trastuzumab or observation (Table 2).

Including the behavior of CETC into the Kaplan-Meier analysis allowed for a clear separation of patients with highly variable or increasing CETC with inferior prognosis from those with decreasing or no change in CETC with a good prognosis in all treatment groups. In Fig. 4, the
(B)

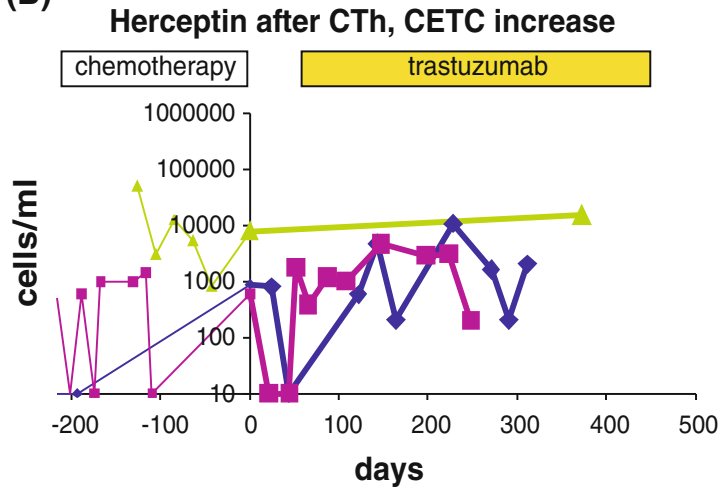

(D)

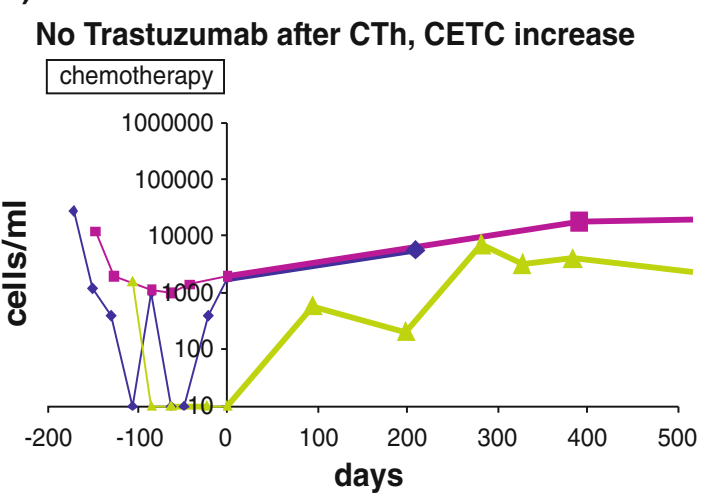

(F)

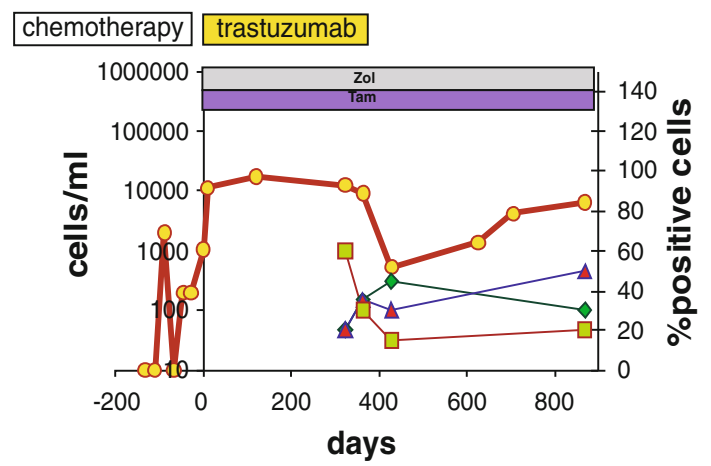

maintenance therapy with trastuzumab CETC. $\mathbf{c}$, d Typical CETC courses in patients without trastuzumab treatment. All three of the patients with increasing CETC have already relapsed. e, f Two patients with decrease as well as increase in CETC during trastuzumab and increase or decrease in the fraction of cells with different amplificates of the HER2/neu gene. The fraction of cells with different amplificates of the HER2/neu gene is given on the left $y$-axis

behavior of CETC in the control group (dashed lines) and in the trastuzumab group (thin lines) is compared for relapse-free survival. In both groups, patients with a decrease in CETC had a highly significantly better cumulative survival (green lines) than patients with increasing CETC (red lines). Even without trastuzumab treatment, a decrease in CETC was associated with excellent survival, 
Table 2 Outcome of patients with decreasing and increasing/highly variable numbers of CETC during maintenance therapy with trastuzumab or observation

\begin{tabular}{|c|c|c|c|c|}
\hline & Total & Relapse & Complete remission & $P$ \\
\hline \multicolumn{5}{|c|}{ No trastuzumab } \\
\hline Decrease & 19 & 4 & 15 & \multirow[t]{2}{*}{0.004} \\
\hline Increase & 9 & 7 & 2 & \\
\hline \multicolumn{5}{|c|}{ Trastuzumab } \\
\hline Decrease & 25 & 1 & 24 & \multirow[t]{2}{*}{$<0.001$} \\
\hline Increase & 11 & 7 & 4 & \\
\hline
\end{tabular}

whereas most patients with highly variable or an increase in CETC would eventually suffer a relapse. The same was true for the group receiving trastuzumab, where all relapsing patients except one were in the group with an increase in CETC, and this was obvious in spite of the relatively short observation time. This was also true for progress in the primarily metastasized patients (dotted lines). This difference in the behavior of CETC (increase or decrease) was independent of lymph node involvement both in patients without and with trastuzumab treatment. The survival curves for patients with increasing and

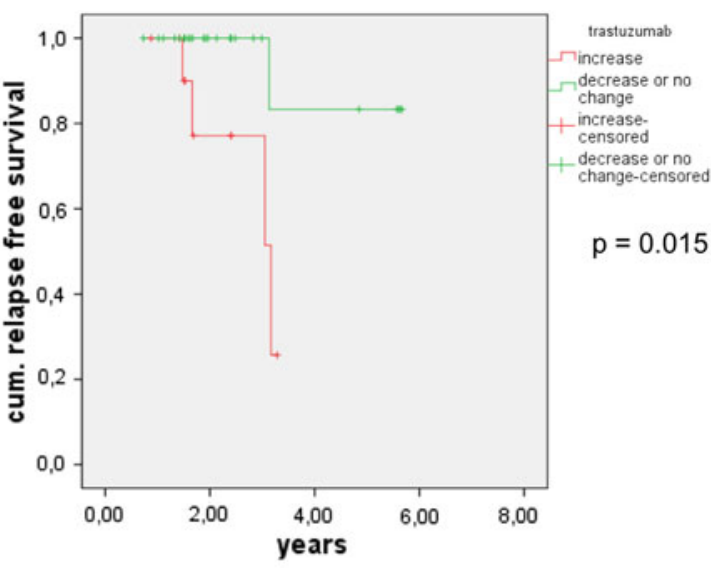

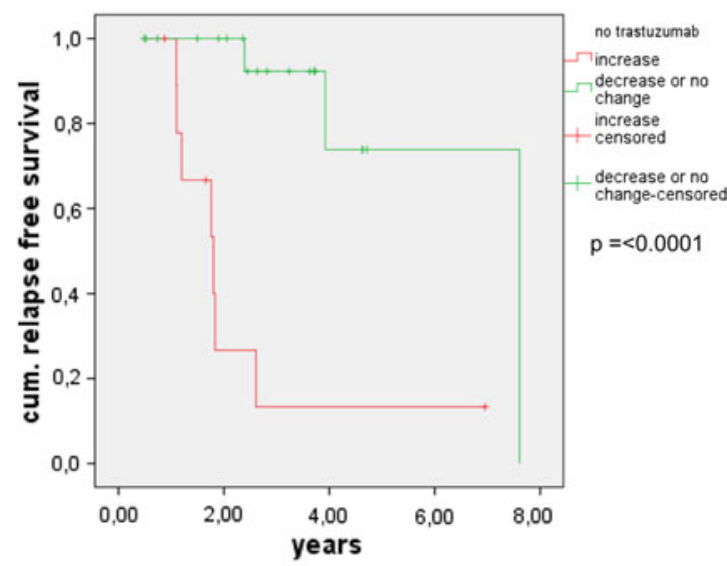

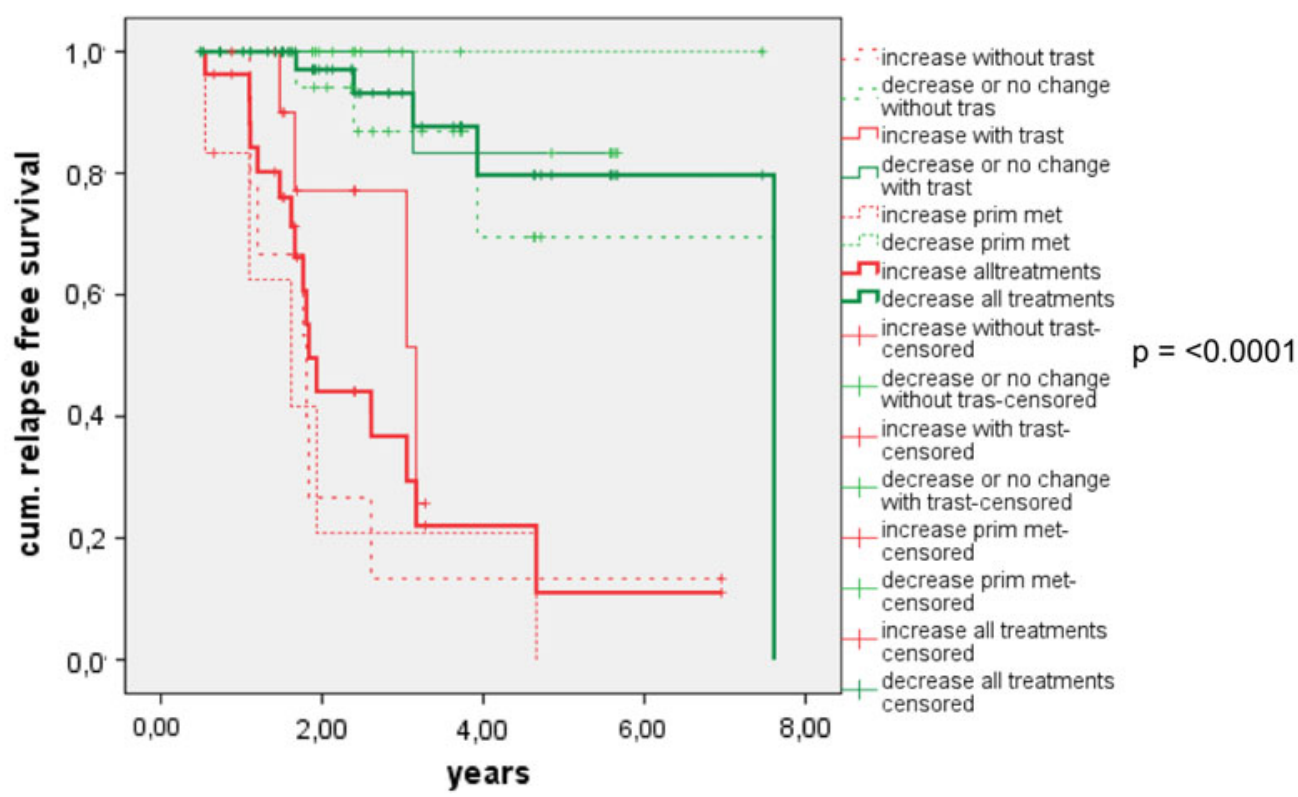

Fig. 4 a Kaplan-Meier relapse-free survival curves for patients without trastuzumab (green line decreasing/stable CETC, red line increasing/variable CETC). b Kaplan-Meier relapse-free survival curves for patients with trastuzumab (green line decreasing/stable CETC, red line increasing/variable CETC). c Combined KaplanMeier relapse-free survival curves for patients with increasing and decreasing CETC in the untreated, the trastuzumab-treated and the primarily metastasized patients receiving trastuzumab together with chemotherapy (dashed lines patients without trastuzumab treatment; red line patients with increasing or variable CETC numbers; green line patients with decreasing or stable CETC numbers). Solid lines patients with trastuzumab treatment; red line patients with increasing or variable CETC numbers; green line patients with decreasing or stable CETC numbers. Dotted lines primarily metastasized patients; red line patients with increasing or variable CETC numbers; green line patients with decreasing or stable CETC numbers. Fat lines all patients combined independent of treatment; red line patients with increasing or variable CETC numbers; green line patients with decreasing or stable CETC numbers 
decreasing CETC in the untreated, the trastuzumab treated, and the primarily metastasized patients are combined in the fat lines demonstrating a highly significant separation between poor and good prognosis patients in all three groups according to the behavior of CETC, independent of disease stage and treatment. In a cox regression analysis, joint analysis of CETC, nodal status, tumor size, and ER status resulted in a regression model in which only CETC behavior significantly influenced the relapse-free survival.

Thus, the response of CETC to therapy makes it possible to distinguish not only between groups, but also individual patients with different outcomes already during therapy.

\section{Discussion}

Based on the results of three studies, trastuzumab therapy is now included into the 2008 ASCO/CAP guidelines as routine therapy and nearly all HER2/neu-positive patients are treated with trastuzumab today (Joensuu et al. 2006; Piccart-Gebhart 2006; Romond et al. 2005; Slamon et al. 2006). Therefore, it was only possible to compare a historical group of control patients not treated with trastuzumab with the trastuzumab-treated patients; a trend to better relapse-free survival in patients treated with trastuzumab was also observed in the patients from our institution, which after four and a half years was, however, no longer statistically significant. This might be due to the relatively low number of patients, but it is obvious that for the trastuzumab-treated patients, the relapse-free survival curve is shifted to the right during the first 3 years and then declines. This indicates that the benefit from 1 year of trastuzumab treatment seems to last mainly for the first 3 years. Since also some of the patients with HER2/neupositive breast cancers before the era of trastuzumab seemed to fare well, it would be desirable to analyze more closely which patients benefit from trastuzumab. This may no longer be possible when in the future all HER2/neupositive patients will receive trastuzumab therapy and even node-negative HER2/neu-positive grade 1 or 2 cancers (Tovey et al. 2009) are no longer classified as "low risk".

In our HER2/neu-positive patients in context with trastuzumab treatment, the difference between lymph nodenegative and -positive patients was not significant. Both lymph node-negative and -positive patients benefited from trastuzumab. The shift to later relapse in patients receiving trastuzumab indicated that relapse was postponed but not averted.

In the primarily metastasized patients, $5 / 8$ patients progressed in spite of simultaneous chemotherapeutic and trastuzumab treatment. The poor outcome of this group might illustrate the limited efficiency of chemotherapy in the presence of trastuzumab. If it is assumed that trastuzumab drives the cells into a resting stage, this would impair the effect of chemotherapy, which is directed preferentially to growing cells, and this might be the cause of a negative interaction with cell cycle-dependent chemotherapy (Lenferink et al. 2001). Such interactions might be the reason for "combinations" of biologically based targeted and traditional chemotherapeutic leading to both successes and failures (Peng et al. 1996; Ling et al. 2007; Vogel and Tan-Chiu 2005). This is also in line with our previous results that have shown that during neoadjuvant treatment sequential trastuzumab therapy leads to better outcome than simultaneous treatment (Gajda et al. 2008). The unexpectedly long survival of one primarily metastasized patient during long-time trastuzumab therapy without further disease progression is in line with the results of Livingstone and Johnstone, showing long-term responses of a fraction of metastasized HER2/neu-positive patients to trastuzumab (Livingston et al. 2008; Johnston et al. 2009).

The routine use of biomarkers is not recommended for early breast cancer (Harris et al. 2007) although it would, undoubtedly, be most beneficial for this group of patients to have a tool to control response to therapy and, ultimately, to prevent the spread of disease by adjusting the treatment. We have previously shown that monitoring CETC during adjuvant chemotherapy can contribute to this goal and may in the future help to improve therapeutic results (Pachmann et al. 2008; Camara et al. 2009).

Having prospectively monitored patients for CETC during the following observation period or during maintenance treatment with trastuzumab since 2001, we were able to evaluate the HER2/neu-positive patients for their pattern of CETC behavior and clinical outcome.

In both the observation and the treatment group, we could clearly distinguish between patients whose CETC numbers decreased or remained at constant levels and those whose CETC numbers increased. Grouping together patients with a high variability in CETC numbers and patients with increasing CETC during trastuzumab treatment led to an increase in significance. There was a highly significant difference in relapse-free survival between these two populations in the observation only group as well as in the trastuzumab treatment group. Patients receiving trastuzumab, who had decreasing or stable CETC, had an excellent relapse-free survival, and only one relapse has occurred during a follow-up time of almost 6 years. This was true also for patients without trastuzumab therapy with decreasing CETC during the 8 years of observation.

The population correlating best with the behavior of total CETC was the group with a high amplification of HER2/neu, indicating that alternating CETC numbers may be due to repeated elimination of CETC by trastuzumab and either re-growth or reseeding possibly from micrometastatic loci. In such cases, it might, in the future, be 
meaningful to increase the trastuzumab dose or add lapatinib. The effect on the CETC might then be able to tell us whether this approach is successful. The behavior of the CETC indicates that there is a biological diversity even in HER/2neu-positive disease that warrants further studies. Indeed, monitoring of the response of CETC to trastuzumab will provide new insights into the nature and behavior of these cells (Brockhoff et al. (2007). CETC monitoring may also help identify biological changes of HER2/neu status in the process of tumor progression (Fehm et al. 2007).

On the other hand, not only patients with decreasing CETC but also with stable CETC had an improved prognosis. This is compatible with results from Brockhoff et al. 2007 who observed a reduced fraction of proliferating cells and prolongation of the G1 phase in vitro and an increase in quiescent cells but no appreciable amount of apoptosis during treatment of cell line cells with trastuzumab. Anti-EGFR agents such as cetuximab, erlotinib, as well as trastuzumab have been shown to induce G1-0 arrest in treated cancer cells (Staal et al. 2009). Driving tumor cells into quiescence may prohibit them from forming metastases without eliminating these cells. Such cells can, however, possibly be reactivated even and this may be the reason why relapses can occur even after years.

We here show that monitoring CETC can help identify patients at high risk for progression already during treatment early on during the course of the disease and, moreover, that monitoring of the response of CETC to trastuzumab will provide new insights into the nature and behavior of these cells.

CETC monitoring might enable patients to be distinguished who farewell without trastuzumab treatment, which may be especially valuable for patients with cardiovascular risk factors. In such cases, it might be considered to reserve trastuzumab therapy as long as the tumor cells remain constant. On the other hand, an increase in or highly unstable CETC even during trastuzumab treatment did signal a significantly impaired outcome. Therefore, it will become possible already during trastuzumab treatment to get hints about therapy success or failure and this may help to change and improve therapy.

\section{Conclusion}

Thus, although the debate with respect to the "true" number of tumor cells in the circulation is still not resolved, we were able to show that the response of the circulating cells was highly significantly correlated to outcome with a high hazard ratio (Sobrero and Bruzzi 2009) permitting the number of patients included in a study to be kept low and this may, in the future, also help to reduce the size of studies necessary to determine treatment success.

The behavior of CETC detected in our approach reliably mirrors the response of the remnant tumor burden to trastuzumab maintenance therapy and may, therefore, be applicable as a biomarker for monitoring the efficacy of targeted therapy in the individual patient.

"Liquid biopsies" of the remnant tumor burden may initiate a paradigm shift because analysis of the systemic part of the tumor allows determining already during therapy whether the respective treatment is effective in the individual patient and in which patients treatment should be changed or complemented. This will reduce lengthy randomized studies that are able to tell only with a statistical probability and after years whether a compound is active and thus help to save costs for the pharmaceutical industry and the health system.

Acknowledgments The authors wish to thank all of the patients and their families who have contributed to this study. The expert assistance of Dr. U. Hammer in analyzing the clinical data is gratefully acknowledged.

Open Access This article is distributed under the terms of the Creative Commons Attribution Noncommercial License which permits any noncommercial use, distribution, and reproduction in any medium, provided the original author(s) and source are credited.

\section{References}

Brockhoff G, Heckel B, Schmidt-Bruecken E, Plander M, Hofstaeder $F$ et al (2007) Differential impact of cetuximab, pertuzumab and trastuzumab on BT474 and SK-BR-3 breast cancer cell proliferation. Cell Prolif 40:488-507

Camara O, Rengsberger M, Egbe A, Koch A, Gajda M et al (2007) The relevance of circulating epithelial tumor cells (CETC) for therapy monitoring during neoadjuvant (primary systemic) chemotherapy in breast cancer. Ann Oncol 18:1484-1492

Camara O, Jörke C, Hammer U, Egbe A, Rabenstein C et al (2009) Monitoring circulating epithelial tumour cells (CETC) to gauge therapy: in patients with disease progression after trastuzumab: persisting CETC can be eliminated by combined lapatinib treatment. J Cancer Res Clin Oncol 135:643-647

Coumans FA, Doggen CJ, Attard G, de Bono JS, Terstappen LW (2010) All circulating EpCAM+CK+CD45-objects predict overall survival in castration-resistant prostate cancer. Ann Oncol 21:1851-1857

Cristofanilli M, Budd GT, Ellis MJ, Stopeck C, Matera J et al (2004) Circulating tumor cells, disease progression, and survival in metastatic breast cancer. N Engl J Med 351:781-791

Darb-Esfahani S, Loibl S, Müller BM, Roller M, Denkert C et al (2009) Identification of biology-based breast cancer types with distinct predictive and prognostic features: role of steroid hormone and HER2 receptor expression in patients treated with neoadjuvant anthracycline/taxane-based chemotherapy. Breast Cancer Res 11:R69

Fehm T, Becker S, Duerr-Stoerzer S, Sotlar K, Mueller V et al (2007) Determination of HER2 status using both serum HER2 levels and circulating tumor cells in patients with recurrent breast 
cancer whose primary tumor was HER2 negative or of unknown HER2 status. Breast Cancer Res 9:R74

Gajda M, Camara O, Oppel S, Kroll T, Joerke C et al (2008) Monitoring circulating epithelial tumor cells (CETC) during primary systemic chemotherapy including trastuzumab for early prediction of outcome in patients with HER2/neu-positive tumors. Ann Oncol 19:2090-2091

Harris L, Fritsche H, Mennel R, Norton L, Ravdin P et al (2007) American Society of Clinical Oncology 2007 update of recommendations for their use of tumor markers in breast cancer. J Clin Oncol 25:5287-5312

Hudis CA (2007) Trastuzumab- mechanism of action and use in clinical practice. N Engl J Med 357:39-51

Joensuu H, Kellokumpu-Lehtinen PL, Bono P, Alanko T, Kataja V et al (2006) Adjuvant docetaxel or vinorelbine with or without trastuzumab for breast cancer. N Engl J Med 354:809-820

Johnston S, O'Rouke L, Wang W et al (2009) Baseline serum extracellular domain HER2 expression in hormone recpetorpositive metastatic breast cancer: correlation with known tissue HER2 status. Cancer Res 69:252s (suppl; abstr 3147)

Lenferink AE, Busse D, Flanagan WM, Yakes FM, Arteaga CL (2001) ErbB2/neu kinase modulates cellular p27(Kip1) and cyclin D1 through multiple signaling pathways. Cancer Res 61:6583-6591

Ling YH, Li T, Yuan Z, Haigentz M Jr, Weber TK et al (2007) Erlotinib, an effective epidermal growth factor receptor tyrosine kinase inhibitor, induces p27KIP1 up-regulation and nuclear translocation in association with cell growth inhibition and G1. Mol Pharmacol 72:248-258

Livingston RB, Downey L, Di Leo A et al (2008) Evaluation of chromosome 17 (Chr-17) polysomy in HER2 FISH-negative metastatic breast cancer (MBC) patients enrolled in a randomized phase III study of paclitaxel and lapatinib. J Clin Oncol 26:42s (suppl; abstr 1006)

Lobodasch K, Fröhlich F, Rengsberger M, Schubert R, Dengler R et al (2007) Quantification of circulating tumor cells for monitoring of adjuvant therapy in breast cancer: an increase in cell number at completion of therapy is a predictor of early relapse. Breast 16:211-218

O'Donovan N, Crown J (2007) EGFR and HER-2 antagonists in breast cancer. Anticancer Res 27:1285-1294

Olopade OI, Grushko TA, Nanda R, Huo D (2008) Advances in breast cancer: pathways to personalized medicine. Clin Cancer 24:7988-7999

Pachmann K, Heiss P, Demel U, Tilz G (2001) Detection and quantification of small numbers of circulating tumour cells in peripheral blood using laser scanning cytometer (LSC). Clin Chem Lab Med 39:811-817

Pachmann K, Clement JH, Schneider CP, Willen B, Camara O et al (2005a) Standardized quantification of circulating peripheral tumor cells from lung and breast cancer. Clin Chem Lab Med 43:617-627

Pachmann K, Camara O, Kavallaris A, Schneider U, Schünemann S et al (2005b) Quantification of the response of circulating epithelial cells to neodadjuvant treatment for breast cancer: a new tool for therapy monitoring. Breast Cancer Res 7:R975R979

Pachmann K, Camara O, Kavallaris A, Krauspe S, Malarski N et al (2008) Monitoring the response of circulating epithelial tumor cells to adjuvant chemotherapy in breast cancer allows detection of patients at risk of early relapse. J Clin Oncol 26:1208-1215

Peng D, Fan Z, Lu Y, DeBlasio E, Scher H et al (1996) Antiepidermal growth factor receptor monoclonal antibody 225 upregulates p27KIP1 and induces G1 arrest in prostatic cancer cell line DU145. Cancer Res 56:3666-3669

Piccart-Gebhart MJ (2006) Adjuvant trastuzumab therapy for HER2overexpressing breast cancer: what we know and what we still need to learn. Eur J Cancer 42:1715-1719

Piccart-Gebhart MJ, Procter M, Leyland-Jones B, Goldhirsch A, Untch $\mathrm{M}$ et al (2005) Trastuzumab after adjuvant chemotherapy in HER2-positive breast cancer. N Engl J Med 353:1659-1672

Romond EH, Perez EA, Bryant J, Suman VJ, Geyer CE Jr et al (2005) Trastuzumab plus adjuvant chemotherapy for operable HER2positive breast cancer. N Engl J Med 353:1673-1684

Slamon DJ, Romond EH, Perez EA (2006) Advances in adjuvant therapy for breast cancer. Clin Adv Hematol Oncol 4(suppl 1):4-9

Sobrero A, Bruzzi P (2009) Incremental advance or seismic shift? The need to raise the bar of efficacy for drug approval. J Clin Oncol 27:5868-5873

Spielmann M, Roché H, Delozier T, Canon JL, Romieu G et al (2009) Trastuzumab for patients with axillary-node-positive breast cancer: results of the FNCLCC-PACS 04 trial. J Clin Oncol 27:6129-6134

Staal S, O'Connell MJ, Allegra CJ (2009) The marriage of growth factor inhibitors and chemotherapy: bliss or bust? J Clin Oncol 27:1545-1548

Tovey SM, Brown S, Doughty JC, Mallon EA, Cooke TE et al (2009) Poor survival outcomes in HER2-positive breast cancer patients with low-grade, node-negative tumours. $\mathrm{Br} \mathrm{J}$ Cancer 100:680-683

Vogel CL, Tan-Chiu E (2005) Trastuzumab plus chemotherapy: convincing survival benefit or not? J Clin Oncol 23:4247-4250 